\title{
Physicochemical, textural and viscoelastic properties of palm diacylglycerol bakery shortening during storage
}

\begin{abstract}
BACKGROUND: Diacylglycerol (DAG), which has health-enhancing properties, is sometimes added to bakery shortening to produce baked products with enhanced physical functionality. Nevertheless, the quantity present is often too little to exert any positive healthful effects. This research aimed to produce bakery shortenings containing significant amounts of palm diacyglycerol (PDG). Physicochemical, textural and viscoelastic properties of the PDG bakery shortenings during 3 months storage were evaluated and compared with those of commercial bakery shortening (CS). RESULTS: PDG bakery shortenings (DS55, DS64 and DS73) had less significant increments in slip melting point (SMP), solid fat content (SFC) and hardness during storage as compared to CS. Unlike CS, melting behaviour and viscoelastic properties of PDG bakery shortenings remained unchanged during storage. As for polymorphic transformation, CS contained only $\beta$ crystals after 8 weeks of storage. PDG bakery shortenings managed to retard polymorphic transformation for up to 10 weeks of storage in DS55 and 12 weeks of storage in DS64 and DS73. CONCLUSION: PDG bakery shortenings had similar if not better storage stability as compared to CS. This is mainly due to the ability of DAG to retard polymorphic transformation from $\beta^{\prime}$ to $\beta$ crystals. Thus, incorporation of DAG improved physical functionality of bakery shortening. Copyright (C) 2010 Society of Chemical Industry
\end{abstract}

Keyword: Palm diacylglycerol, Palm stearin, Bakery shortening, Physicochemical properties, Textural properties, Viscoelastic properties, Storage properties 